\title{
VAlorisation des COPRoduits de CReVette (Penaeus SPP.) PAR HYDROLYSE ENZYMATIQUE
}

\author{
VAlORIZATION OF SHRIMP BY-PRODUCTS (PENAEUS SPP.) \\ BY ENZYMATIC HYDROLYSIS
}

\section{VAlorización de SubProductos de CAMARONeS (PenAeUS SPP.) POR HIDRÓLISIS ENZIMÁTICA}

\author{
M. Ravoninjatovo ${ }^{1 *}$ Z. Randriamahatody ${ }^{1}$ C. Ravonizafy ${ }^{1}$ B. Ramananjaona ${ }^{1}$ \\ M. Rajaonarivony ${ }^{1} \mathrm{H}$. Randrianatoro ${ }^{1}$ A. Rajoelisoa ${ }^{1}$
}

\author{
Mots-clés : Crevette - Sous-produit - Hydrolyse enzymatique - \\ Pepsine - Propriété physicochimique - Madagascar. \\ Keywords: Prawns and shrimps - By-product - Enzymatic \\ hydrolysis - Pepsin - Chemicophysical property - Madagascar. \\ Palabras clave: Gambas y camarones - Subproducto - \\ Hidrólisis enzimática - Pepsina - Propiedad \\ físicoquímica - Madagascar.
}

\begin{abstract}
A Madagascar, la production halieutique annuelle est estimée à 150000 tonnes. La crevette occupe une place importante dans l'économie malgache et constitue 73 p. 100 des exportations de produits halieutiques (2). Cependant, une grande partie des produits entiers n'est pas destinée à la consommation humaine, étant constituée essentiellement par les carapaces et les têtes dont l'élimination peut poser un problème pour l'environnement. Pourtant, ces déchets contiennent des composants valorisables, notamment des protéines, des lipides et des minéraux, pour l'amélioration de l'alimentation humaine. L'objectif de cette étude a été d'extraire par hydrolyse enzymatique les composés contenus dans les coproduits de crevettes, de les caractériser et de déterminer les propriétés fonctionnelles des fractions obtenues après hydrolyse.
\end{abstract}

Pour l'hydrolyse, le processus décrit dans une étude antérieure (3) a été adopté avec quelques modifications : les carapaces et les têtes de crevettes ont été hydrolysées en présence de 2 p. 100 de pepsine pendant $2 \mathrm{~h}$ à $37^{\circ} \mathrm{C}$ et à $\mathrm{pH} 2$. Cette hydrolyse a permis d'obtenir trois fractions : le surnageant, l'eau de lavage du culot, et les résidus de lavage après inactivation de l'enzyme par neutralisation du milieu, centrifugation et lavage. Ces fractions ont été caractérisées (matières sèches, protéines, lipides et cendres brutes) et les propriétés fonctionnelles (propriété moussante, propriété d'absorption de lipides et propriété d'adsorption d'eau) du surnageant et de l'eau de lavage du culot ont été déterminées.

Pour la détermination de la propriété moussante, $20 \mathrm{ml}$ de la solution préparée à 1 p. 100 de l'échantillon ont été homogénéisés

\footnotetext{
1. Centre national de recherches sur l'environnement, 39 rue Rasamimanana Fiadanana, BP 1739, Antananarivo 101, Madagascar.

* Auteur pour la correspondance

E-mail : rmboahangy@gmail.com
}

à $9500 \mathrm{tr} / \mathrm{min}$ pendant $1 \mathrm{~min}$. Le volume de la mousse formée a été mesuré à $0,30 \mathrm{~s}$, et à 5, 10, 40 et $60 \mathrm{~min}$. La capacité moussante a été exprimée par le pourcentage de l'augmentation du volume de la mousse à 0 min tandis que la stabilité de la mousse a été exprimée par l'expansion de la mousse durant 60 min $(1,5)$. La propriété d'absorption de lipides a été obtenue par la détermination du volume d'huile absorbé par gramme de protéine après homogénéisation de 500 mg d'échantillon et $10 \mathrm{ml}$ d'huile, avec agitation toutes les $10 \mathrm{~min}$ pendant $30 \mathrm{~min}$ et centrifugation à $2500 \mathrm{tr} / \mathrm{min}$ pendant $25 \mathrm{~min}(1,4)$. La propriété d'adsorption d'eau a été obtenue par la détermination du volume d'eau absorbé par gramme de protéine après homogénéisation de $500 \mathrm{mg}$ d'échantillon et $10 \mathrm{ml}$ d'eau avec agitation toutes les $10 \mathrm{~min}$ pendant $30 \mathrm{~min}$ et centrifugation à $2500 \mathrm{tr} / \mathrm{min}$ pendant $25 \min (4)$.

Les têtes de crevettes sont riches en protéines et les carapaces contiennent une quantité considérable de cendres brutes. Après hydrolyse pepsique, le taux $d^{\prime}$ 'hydrolyse des carapaces (57 p. 100) a été plus élevé que celui des têtes de crevettes (48 p. 100) (figure 1). Les résultats de la caractérisation des fractions obtenues ont montré que le surnageant contenait une quantité importante de protéines et présentait une propriété moussante intéressante (figures 2 et 3). En effet, I'hydrolyse enzymatique a donné lieu à des protéines de plus petite taille, augmentant leur solubilité et donc leur passage dans la phase soluble. Les résidus de lavage ont représenté une fraction chitineuse pauvre en cendres (tableau I). Le culot a montré une propriété d'absorption de lipides et d'adsorption d'eau non négligeable qui mérite d'être valorisée (figures 3 et 4).

L'hydrolyse des coproduits de crevettes avec la pepsine a permis de séparer diverses molécules dans plusieurs fractions dont certaines possèdent des propriétés fonctionnelles intéressantes. Elle peut ainsi constituer une voie de valorisation permettant de récupérer différentes molécules d'intérêt.

Actuellement, des études sur la toxicité des produits sont effectuées et leur utilisation en alimentation est envisagée. En outre, des études sur la composition des acides aminés des protéines du surnageant des carapaces et des têtes de crevettes, ainsi que sur la composition des acides gras contenus dans les lipides du culot sont envisagées pour la valorisation de chaque fraction issue de l'hydrolyse. 


\section{Tableau I}

Composition biochimique des fractions obtenues après deux heures d'hydrolyse pepsique

\begin{tabular}{|c|c|c|c|c|c|c|}
\hline \multirow[b]{3}{*}{ Paramètres } & \multicolumn{6}{|c|}{ Composition moyenne (g / 100 g de MS \pm écart-type) } \\
\hline & \multicolumn{3}{|c|}{ Têtes } & \multicolumn{3}{|c|}{ Carapaces } \\
\hline & Surnageant & Culot & Résidus & Surnageant & Culot & Résidus \\
\hline Protéines totales & $48,85 \pm 1,28$ & $30,55 \pm 0,89$ & $28,63 \pm 0,52$ & $47,92 \pm 0,44$ & $32,91 \pm 0,09$ & $22,86 \pm 0,98$ \\
\hline Lipides totaux & $1,96 \pm 0,01$ & $16,90 \pm 0,96$ & $1,40 \pm 0,06$ & $0,55 \pm 0,18$ & $3,10 \pm 0,74$ & $1,24 \pm 0,18$ \\
\hline Cendres brutes & $43,80 \pm 1,37$ & $35,64 \pm 0,00$ & $9,77 \pm 0,07$ & $47,91 \pm 0,51$ & $52,53 \pm 0,61$ & $2,74 \pm 0,13$ \\
\hline
\end{tabular}

MS : matière sèche

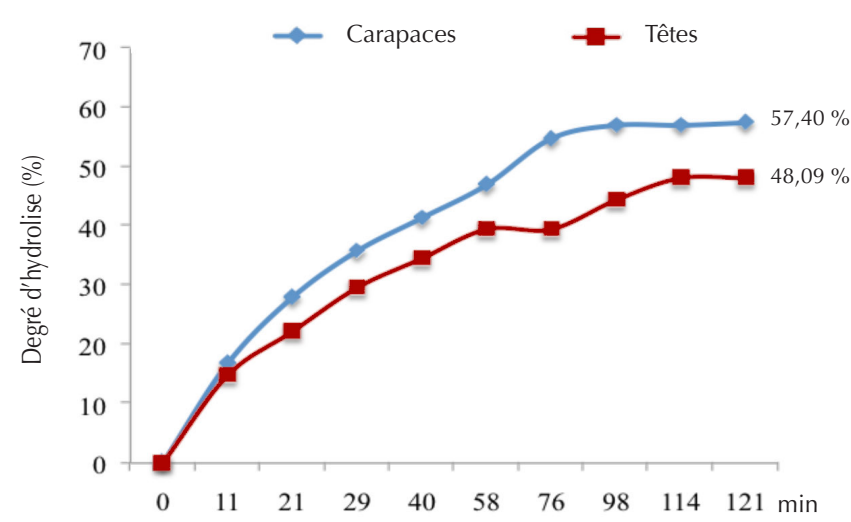

Figure 1 : hydrolyse enzymatique des têtes et des carapaces de crevettes avec la pepsine $2 \%$.

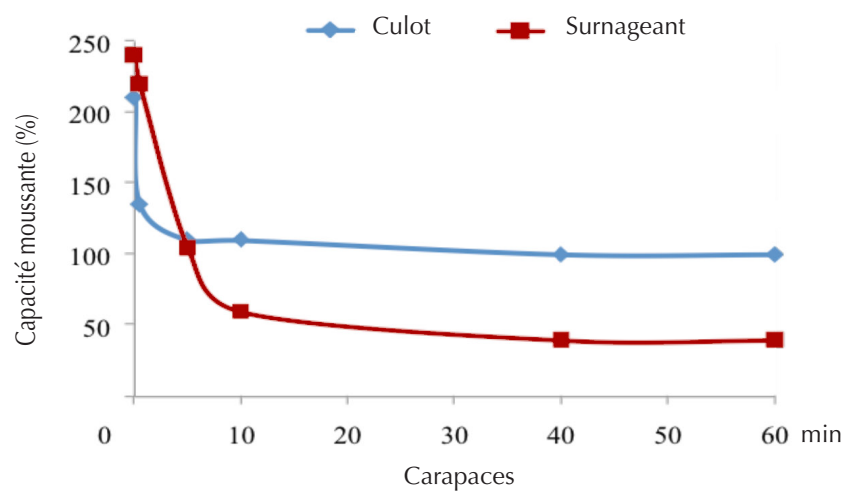

Figure 2 : capacité moussante du culot et du surnageant des carapaces de crevettes.

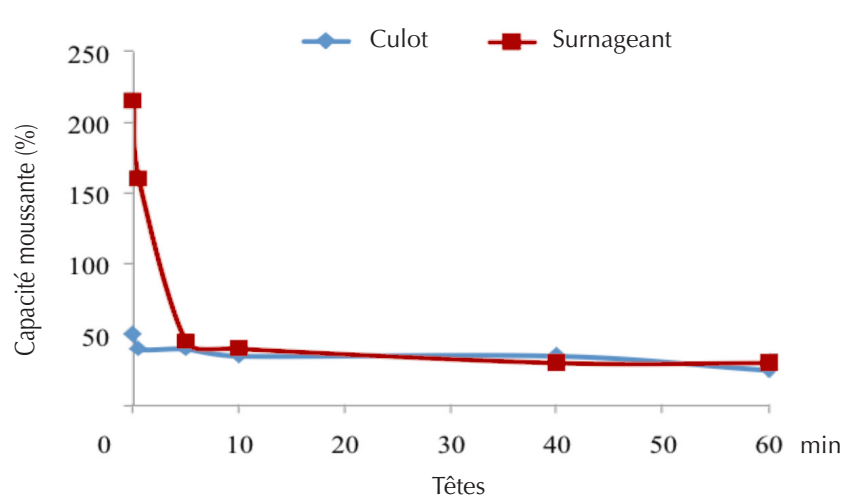

Figure 3 : capacité moussante du culot et du surnageant des têtes de crevettes.

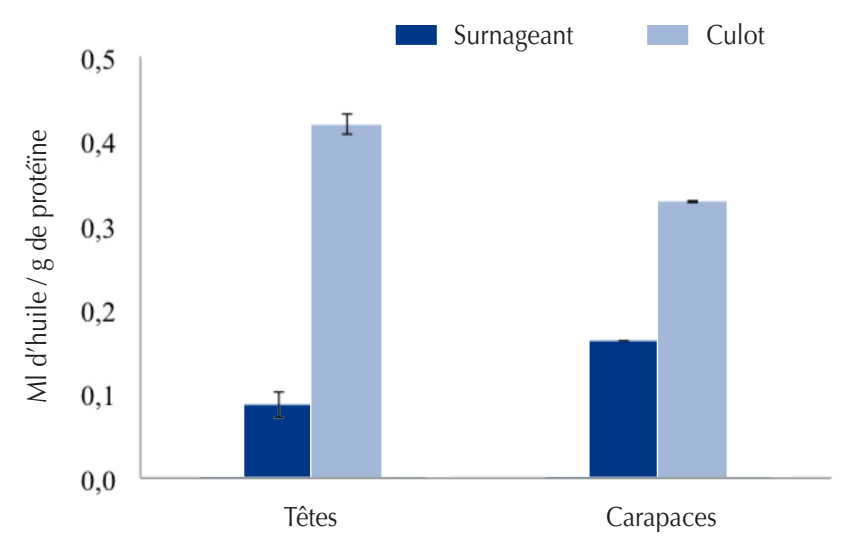

Figure 4 : propriété d'absorption de lipides du surnageant et du culot des carapaces et des têtes de crevettes.

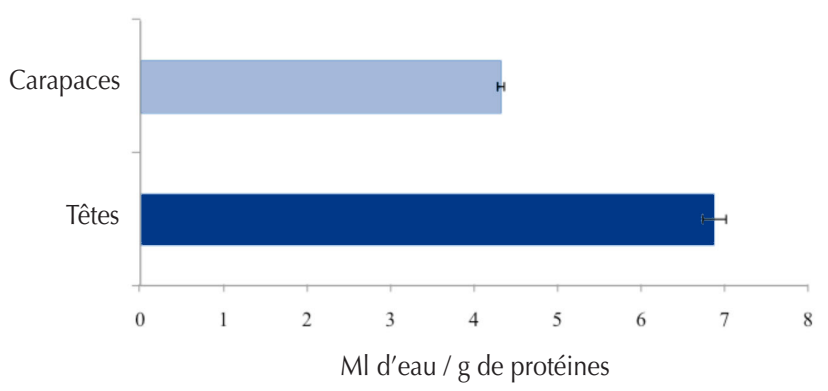

Figure 5 : propriété d'adsorption d'eau du culot des carapaces et des têtes de crevettes.

\section{BIBLIOGRAPHIE}

1. LIMAM Z., SADOK S., EL ABED A., 2008. Enzymatic hydrolysis of shrimp head waste: functional and biochemical properties. Food Biotechnol., 22: 352362.

2. MISSION ECONOMIQUE DE TANANARIVE, 2008. Le secteur halieutique à Madagascar. UBIFRANCE, $4 \mathrm{p}$.

3. RANDRIAMAHATODY Z., SYLLA K.S.B., NGUYEN H.T.M., DONNAYMORENO C., RAZANAMPARANY L., BOURGOUGNON N., BERGE J.P., 2011. Proteolysis of shrimp by-products (Penaeus monodon) from Madagascar. CYTA - J. Food, 9: 220-228. DOI: 10.1080/19476337.2010.518250

4. SATHIVEL S., SMILEY S., PRINYAWIWATKULW., BECHTEL P.J., 2005. Functional and nutritional properties of red salmon (Oncorhynchus nerka) enzymatic hydrolysates. J. Food Sci., 70: c401-c406.

5. THIANSILAKUL Y., BENJAKUL S., SHAHIDI F., 2006. Composition, functional properties and antioxidative activity of protein hydrolysates prepared from round scad (Decapterus maruadsi). Food Chem., 103: 1385-1394.

Accepted 30 April 2015; Online publication June 2015 